Pacific Journal of Mathematics

STRUCTURE OF NOETHER LATTICES WITH 


\title{
STRUCTURE OF NOETHER LATTICES WITH JOIN-PRINCIPAL MAXIMAL ELEMENTS
}

\author{
E. W. Johnson AND J. P. LediaeV
}

\section{In this paper we explore the structure of Noether lattices with join-principal maximal elements.}

Results which completely specify the structure of certain special classes of Noether lattices, and relate them to lattices of ideals of Noetherian rings, have been obtained in [1], [2], [3], [4], [7], and [8]. For example, in [7] we showed that if every maximal element of a Noether lattice $\mathscr{L}$ is meet-principal, then $\mathscr{L}$ is distributive and can be represented as the lattice of ideals of a Noetherian ring. Moreover, for distributive Noether lattices, the condition that every maximal element is meet-principal is equivalent to representability. In a more recent paper [8], we began considering the complementary case of a Noether lattice in which every maximal element is join-principal in order to determine the extent of the relationship between the two situations. There we showed that if 0 is prime in $\mathscr{L}$ (and every maximal element is join-principal), then $\mathscr{L}$ is distributive and representable. Hence, if 0 is prime, the assumptions that every maximal element is meet-principal and that every maximal element is joinprincipal are equivalent, and either implies representability.

In this paper, we continue the investigation begun in [8]. Our results extend the class of Noether lattices for which embedding and structure theorems are known, and also introduce a construction process for Noether lattices which leads to new examples.

In $\S 1$, we show that in a local Noether lattice $(\mathscr{L}, M)$ in which $M$ is join-principal and not a prime of 0 , the maximal element $M$ has a minimal base $E_{1}, \cdots, E_{k}$ of independent principal elements (i.e., $E_{i} \wedge\left(E_{1} \vee \cdots \vee \hat{E}_{i} \vee \cdots \vee E_{k}\right)=0$ for $\left.i=1, \cdots, k\right)$. And we use this result to show that if $M$ is join-principal and not a prime of 0 , then $\mathscr{L}$ is distributive. In $\S 2$, we obtain structure and embedding theorems for distributive local Noether lattices with join-principal maximal elements. In $\S 3$, we investigate some of the consequences of our results outside of the local case.

We adopt the terminology of [5].

1. Let $(\mathscr{L}, M)$ be a local Noether lattice and let $B \in \mathscr{L}$. The quotient $B / M B$ is a finite dimensional complemented modular lattice and the number of elements in any minimal set of principal elements with join $B$ is the dimension of the quotient $B / M B([4],[6])$. Hence 
if $E_{1}, \cdots, E_{s}$ is any set of principal elements with the property that the elements $E_{i} \vee M B$ are independent in $B / M B$, then $E_{1}, \cdots, E_{s}$ can be extended to a minimal base for $B$. We will have occasion to use these observations in what follows.

In this section we show that if $(\mathscr{L}, M)$ is a local Noether lattice in which $M$ is join-principal and not a prime of 0 , then $\mathscr{L}$ is distributive.

We begin with a lemma.

Lemma 1.1. Let $(\mathscr{L}, M)$ be a local Noether lattice in which $M$ is join-principal and not a prime of 0 . Let $E_{1}, \cdots, E_{k}$ be a minimal base for $M$ and, for each $i=1, \cdots, k$, set $C_{i}=E_{1} \vee \cdots \vee \hat{E}_{i} \vee \cdots \vee E_{k}$. Then each of the elements $C_{i}(i=1, \cdots, k)$ is prime.

Proof. Since $M$ is principal in $\mathscr{L} / C_{i}(i=1, \cdots, k)$, each of the elements $C_{i}$ is either prime or $M$-primary [7]. Assume that $C_{r}$ is $M$-primary. And let $n$ be the least positive integer such that $E_{r}^{n+1} \leqq C_{r}$. Then $E_{r}^{n+1} \leqq M C_{r}$. For, if not, there exist principal elements $F_{1}, \cdots, F_{s}$ among $E_{1}, \cdots, \hat{E}_{r}, \cdots, E_{k}$ such that $E_{r}^{n+1}, F_{1}, \cdots, F_{s}$ is a minimal base for $C_{r}$. But then $E_{r}, F_{1}, \cdots, F_{s}$ is a minimal base for $M=E_{r} \vee C_{r}$. Since $C_{r}$, by definition, has fewer elements in a minimal base than $M$, this is a contradiction. Hence $E_{r}^{n+1} \leqq M C_{r}$, as claimed. Consequently, $M^{n+1} \leqq M C_{r}$, and therefore

$$
E_{r}^{n} \leqq M^{n} \vee(0: M)=M^{n+1}: M=M C_{r}: M=C_{r} \vee(0: M)=C_{r},
$$

since $M$ is join-principal and not a prime of 0 . Since $E_{r}^{n} \not \equiv C_{r}$, this leads to a contradiction. Hence, each of the elements $C_{i}$ is prime.

Lemma 1.2. Let $(\mathscr{L}, M)$ be a local Noether lattice in which $M$ is join-principal and not a prime of 0 . Then, in the notation of Lemma 1.1, $C_{1} \wedge \cdots \wedge C_{k}=0$.

Proof. Let $E_{1}, \cdots, E_{k}$ and $C_{1}, \cdots, C_{k}$ be as in Lemma 1.1. We first show that for $1 \leqq r<s \leqq k, E_{r} E_{s}=0$. Hence, suppose that $E_{r} E_{s} \neq 0$, and let $n$ be a positive integer such that $E_{r} E_{s} \leqq M^{n}$ and $E_{r} E_{s} \geqq M^{n+1}$. Then $E_{r} E_{s}$ can be used in a minimal base for $M^{n}$. Now, since $M$ is join-principal and not a prime of 0 , it follows from the relation $M^{n k+n}=M^{n k}\left(E_{1}^{n} \vee \cdots \vee E_{k}^{n}\right)$ that the elements $E_{1}^{n}, \cdots, E_{k}^{n}$ form a minimal base for $M^{n}$. Hence, for some $i, 1 \leqq i \leqq k, M^{n}=$ $E_{r} E_{s} \vee E_{1}^{n} \vee \cdots \vee \hat{E}_{i}^{n} \vee \cdots \vee E_{k}^{n}$. But then $M^{n} \leqq C_{i}$, which contradicts Lemma 1.1. It now follows that, for each $s(1 \leqq s \leqq k), C_{s} \wedge E_{s}=$ $\left(C_{s}: E_{s}\right) E_{s}=C_{s} E_{s}=0$, since $C_{s}$ is prime and $E_{s} \not C_{s}$. Hence by modularity $C_{1} \wedge \cdots \wedge C_{s}=E_{s+1} \vee \cdots \vee E_{k}$ for $s \leqq k$, so that $C_{1} \wedge \cdots \wedge C_{k}=0$. 
We are now in a position to establish the main result of the section.

Theorem 1.3. Let $(\mathscr{L}, M)$ be a local Noether lattice in which $M$ is a join-principal and not a prime of 0 . Then $\mathscr{L}$ is distributive.

Proof. Let $E_{1}, \cdots E_{k}$ and $C_{1}, \cdots, C_{k}$ be as in Lemma 1.1. A simple inductive argument using modularity proves that

$$
\left(\vee E_{i}^{j(i)}\right) \wedge\left(\vee E_{i}^{k(i)}\right)=\vee E_{i}^{\max (j(i), k(i))}
$$

with the convention that $E_{i}^{\infty}$ means 0 . Thus it suffices to show that the only principal elements in $\mathscr{L}$ are $0, I$ and the powers $E_{i}^{n}$ of the elements $E_{1}, \cdots, E_{k}$. If $k=1$, the result is immediate, so assume $k \geqq 2$. Let $E$ be any principal element of different from 0 and $I$. We assume that the elements $E_{1}, \cdots, E_{k}$ are arranged so that $E \leqq C_{i}$ for $i>r$ and $E \geqq C_{i}$ for $i \leqq r$. Set $C=C_{1} \wedge \cdots \wedge C_{r}$ and consider $\mathscr{L} / C$. Since $M$ is principal in each of the local Noether lattices $\mathscr{L} / C_{i}(i=1, \cdots, k)$, it follows by Lemmas 1.1 and 1.2 that the primes of $\mathscr{L} / C$ are just $M$ and $C_{1}, \cdots, C_{r}$. Hence, by the choice of $E$, the element $E \vee C$ is $M$-primary in $\mathscr{L} / C$, and therefore also in $\mathscr{L}$. Let $n$ be a positive integer such that $M^{n+1} \leqq E^{*} \vee C$ and $M^{n} \not \equiv E \vee C$, then, by modularity,

$$
\begin{aligned}
M^{n+1} \vee C & =C \vee\left(\left(M^{n+1} \vee C\right) \wedge E\right) \\
& =C \vee\left(\left(M^{n+1} \vee C\right): E\right) E .
\end{aligned}
$$

Hence, either $M^{n+1} \leqq C \vee M E$ or $\left(M^{n+1} \vee C\right): E=I$. In the first case, however,

$$
M^{n} \leqq M^{n+1}: M \leqq(C \vee M E): M=(C: M) \vee E=C \vee E,
$$

which contradicts the choice of $n$. Hence $\left(M^{n+1} \vee C\right): E=I$ and $E \leqq M^{n+1} \vee C$. Then $E \vee C=M^{n+1} \vee C$, so by the join-irreducibility of principal elements in a local Noether lattice, it follows that $E \vee C=E_{1}^{o(1)} \cdots E_{i k}^{o(k)} \vee C$, for some nonnegative integers $\varphi(1), \cdots, \varphi(k)$. On the other hand, $E_{i} \leqq C$ for $i>r$ and $E \geqq C$, so $\varphi(i)=0$ for $i>r$. Now, if $i \neq j$ and $1 \leqq j \leqq r$, then $E_{i} \vee C \leqq C_{j}$. It follows that $r \leqq 1$, and hence that $E \leqq C_{2} \wedge \cdots \wedge C_{k}$. Then by the proof of Lemma 1.2, $C_{2} \wedge \cdots \wedge C_{k}=E_{1}$ and $M E_{1}^{n}=E_{1}^{n+1}$, for all $n$. Hence, there exists a positive integer $u$ such that $E \leqq E_{1}^{u}$ and $E \$ M E_{1}^{u}=$ $E_{1}^{u+1}$. Since $E_{1}$ is principal, it is now immediate that $E=E_{1}^{u}$.

We note that if $(\mathscr{L}, M)$ is a local Noether lattice in which $M^{2}=0$, then $M$ is join-principal. Since such a Noether lattice need not be distributive, the statement of Theorem 1.3 need not be valid without the assumption that $M$ is not a prime of 0 . On the other hand, if $\mathscr{L}$ 
is an arbitrary Noether lattice in which every maximal element is join-principal, then the number of maximal primes associated with 0 is finite. Hence, at most finitely many of the localizations $\mathscr{L}_{M}(M$ maximal) are nondistributive.

2. Let $\left(\mathscr{L}_{1}, M_{1}\right)$ and $\left(\mathscr{L}_{2}, M_{2}\right)$ be local Noether lattices, and let $\mathscr{L}=\left\{(A, B) \in \mathscr{L}_{1} \oplus \mathscr{L}_{2} ; A=I\right.$ if and only if $\left.B=I\right\}$. It is clear that $\mathscr{L}$ is a sub-multiplicative-lattice of $\mathscr{L}_{1} \oplus \mathscr{L}_{2}$. Moreover, if $E_{1}$ and $E_{2}$ are principal elements of $\mathscr{L}_{1}$ and $\mathscr{L}_{2}$, respectively, with $E_{1} \neq I$ and $E_{2} \neq I$, then the elements $\left(E_{1}, 0\right)$ and $\left(0, E_{2}\right)$ are principal in $\mathscr{L}$. Hence $\mathscr{L}$ is a local Noether lattice with maximal element $\left(M_{1}, M_{2}\right)$. We refer to $\mathscr{L}$ as the local direct sum of $\mathscr{L}_{1}$ and $\mathscr{L}_{2}$. An alternative characterization is given by $\mathscr{L}=\left(M_{1}\left|0 \oplus M_{2}\right| 0\right) \cup\{(I, I)\}$.

In this section we continue our investigation of a local Noether lattice $(\mathscr{L}, M)$ with join-principal maximal element. However, we drop the hypothesis that $M$ is not a prime of 0 and consider, instead, the general distributive case. Our main result is that a distributive local Noether lattice $(\mathscr{L}, M)$, in which $M$ is join-principal, is the local direct sum of local Noether lattices with principal maximal elements. We begin with an extension of Lemma 1.2.

Lemma 2.1. Let $(\mathscr{L}, M)$ be a distributive local Noether lattice in which $M$ is join-principal. Let $E_{1}, \cdots, E_{k}$ be a minimal base for M. Then $E_{i} \wedge E_{j}=0$ for all $i \neq j$.

Proof. For each $i=1, \cdots, k$, set $C_{i}=E_{1} \vee \cdots \vee \widehat{E}_{i} \vee \cdots \vee E_{k}$. Then

and

$$
M=M^{2}: M=\left(M C_{i} \vee E_{i}^{2}\right): M=C_{i} \vee\left(E_{i}^{2}: M\right)
$$

$$
E_{i} \vee\left(E_{i}^{2}: M\right)=\left(M E_{i} \vee E_{\imath}^{2}\right): M=M E_{i}: M=E_{i} \vee(0: M),
$$

so because

$$
\left(E_{\imath}^{2}: M\right)=\left(E_{i}^{2}: M\right) \wedge\left(E_{i} \vee 0: M\right)=0: M \vee\left(\left(E_{i}^{2}: M\right) \wedge E_{i}\right)
$$

by modularity, we have that

$$
M=C_{i} \vee(0: M) \vee\left(\left(E_{i}^{2}: M\right) \wedge E_{i}\right)=C_{i} \vee(0: M) \vee\left(E_{i}^{2}: M E_{i}\right) E_{i},
$$

$i=1, \cdots, k$. Since principal elements are join-irreducible in a local Noether lattice, since $\mathscr{L}$ is distributive, and since $E_{i} \not \equiv C_{i}$, it follows that either $E_{i} \leqq 0: M$ or $E_{i} \leqq\left(E_{i}^{2}: M E_{i}\right) E_{i}, i=1, \cdots, k$.

Assume that $E_{r} \leqq\left(E_{r}^{2}: M E_{r}\right) E_{r}$. Then $E_{r}^{2}: M E_{r}=I$, so $M E_{r}=E_{r}^{2}$. Hence $M=M E_{r}: E_{r}=E_{r}^{2}: E_{r}=E_{r} \vee\left(0: E_{r}\right)$. It follows that $E_{i} \leqq$ $E_{r} \vee\left(0: E_{r}\right)$ for all $i$, and that $E_{i} \leqq 0: E_{r}$ for $i \neq r$ since $\mathscr{L}$ is distributive and $E_{i}$ is join-irreducible. Therefore $C_{i} E_{i}=0(i=1, \cdots, k)$. 
Now, assume that $1 \leqq i<j \leqq k$ and let $E$ be a principal element such that $E \leqq E_{i} \wedge E_{j}$. Suppose that $E \neq 0$ and choose integers $u$ and $v$ such that $E \leqq E_{1}^{u} \wedge E_{j}^{v}, E \geqq E_{i}^{u+1}$ and $E \geqq E_{j}^{v+1}$. Then $E \leqq E_{\imath}^{u}$ and $E \not M E_{i}^{u}$, so $E=E_{i}^{u}$. Similarly $E=E_{j}^{v}$, so $E_{i}^{u}=E=E_{j}^{v}$. Then $u>1$ and $v>1$, so $M E_{i}^{u-1}=M E_{j}^{v-1}$. It follows that $E_{i}^{u-1} \vee(0: M)=$ $E_{\jmath}^{v-1} \vee(0: M)$, so that either $E_{i}^{u-1} \leqq E_{j}^{v-1}$ or $E_{i}^{u-1} \leqq 0: M$. In either case, $E_{i}^{u}=0$. Hence $E=0$ and $E_{i} \wedge E_{j}=0$.

THeOREM 2.2. Let $(\mathscr{L}, M)$ be a distributive local Noether lattice. Then $M$ is join-principal if, and on!'y if, $\mathscr{L}$ is the (finite) local direct sum of loca? Noether lattices with principal maximal elements.

Proof. Assume that $(\mathscr{L}, M)$ is a distributive local Noether lattice in which $M$ is join-principal. Let $E_{1}, \cdots, E_{k}$ be a minimal base for $M$. And for each $i=1, \cdots, k$, let $\left(\mathscr{L}_{i}, M_{i}\right)$ be a local Noether lattice such that $M_{i}$ is principal and $M_{i}^{n}=0$ if, and only if, $E_{i}^{n}=0$. Since $\mathscr{L}$ is distributive, it follows by Lemma 2.1 and [2] that every element $A \in \mathscr{L}$ has a unique minimal basis consisting of powers of the elements $E_{1}, \cdots, E_{k}$. If we set $E_{\imath}^{\infty}=0$ and $E_{i}^{0}=I$, then it is clear that the map $E_{1}^{n_{1}} \vee \cdots \vee E_{k}^{n_{k}} \rightarrow\left(M_{1}^{n_{1}}, \cdots, M_{k}^{n_{k}}\right)$ is a multiplicative lattice isomorphism of $\mathscr{L}$ onto the local direct sum of $\mathscr{L}_{1}, \cdots, \mathscr{L}_{k}$.

The converse is clear.

Corollary 2.3. Let $(\mathscr{L}, M)$ be a distributive local Noether lattice in which $M$ is join-principal. Then $\mathscr{L}$ is Noether-lattice-embeddable in the lattice of ideals of a homomorphic image of a regular local ring.

Proof. By Corollary 2.2, $\mathscr{L}$ is the local direct sum of local Noether lattices $\left(\mathscr{L}_{1}, M_{1}\right), \cdots,\left(\mathscr{L}_{k}, M_{k}\right)$, where, for each $i, M_{i}$ is principal in $\mathscr{L}_{i}$. If $M_{i}$ is nilpotent in $\mathscr{L}_{i}$, let $n_{i}$ be the least positive integer such that $M_{i}^{n_{i}}=0$; otherwise, let $n_{i}=\infty$. Let $R L_{k}$ be the regular local Noether lattice introduced in [1], and let $X_{1}, \cdots, X_{k}$ be the minimal base for the maximal element of $R L_{k}$. Let $A$ be the join of the elements $X_{i} X_{j}$ and $X_{i}^{n_{i}}$ (where $X_{i}^{\infty}=0$ ). Then $\mathscr{L}$ is clearly isomorphic to $R L_{k} / A$. Since $R L_{k}$ is Noether-lattice-embeddable in the lattice of ideals of a regular local ring, [1], it follows that $R L_{k} / A$ and $\mathscr{L}$ are embeddable in the lattice of ideals of a homomorphic image of a regular local ring.

3. In this section we interpret some of the implications of the results of $\S \S 1$ and 2 outside of the local case.

We begin with a new characterization of the representable distributive Noether lattices. 
Theorem 3.1. Let $\mathscr{L}$ be a Noether lattice. Then $\mathscr{L}$ is distributive and representable as the lattice of ideals of a Noetherian ring if, and only if, for each maximal element $M$ of $\mathscr{L}, M$ is join-principal and $O_{M}$ is meet-irreducible.

Proof. If $\mathscr{L}$ is distributive and representable, then each maximal element $M$ is principal [7]. Consequently, $\mathscr{L}_{M}$ is a quotient of a regular local Noether lattice of altitude 1 , and $O_{M}$ is meet-irreducible.

Now, assume that $\mathscr{L}$ is a Noether lattice such that, for every maximal element $M, M$ is join-principal and $O_{M}$ is meet-irreducible. Fix $M$ and consider $\mathscr{L}_{M}$. If $\{M\}$ is not a prime of 0 in $\mathscr{L}_{M}$, then by Lemma 2.1, $O_{M}$ is meet-irreducible if, and only if, $\{M\}$ is principal. On the other hand, if $\{M\}$ is a prime of 0 in $\mathscr{L}_{M}$, then $\{M\}$ is the only prime of 0 . In this case, let $E$ be any principal element such that $E \leqq 0:\{M\}$. Then $\{M\} E=0$, so $E$ is a point in $\mathscr{L}_{M}$. Since the meet of any two points is 0 and $O_{M}$ is irreducible by assumption, it follows that $0:\{M\}$ is itself a point and that $0:\{M\} \leqq A$, for every $A \neq 0$. Now, assume that $\{M\} \neq 0:\{M\}$, and let $F$ be a principal element such that $F \leqq\{M\}, F \not \leq\{M\}^{2}$ and $\{M\} F \neq 0$. Then $F$ is $\{M\}-$ primary, so there is a nonnegative integer $n$ such that $\{M\}^{n} \not \mid F$ and $\{M\}^{n+1} \leqq F$. Hence $\{M\}^{n+1}=\{M\}^{n+1} \wedge F=\left(\{M\}^{n+1}: F\right) F$, and therefore either $\{M\}^{n+1}: F=I$ or $\{M\}^{n+1} \leqq\{M\} F$. In the first case, $\{M\}^{n+1}=F$, so $\{M\}=F$ by the choice of $F$. In the second case,

$$
\{M\}^{n} \leqq\{M\}^{n+1}:\{M\}=\{M\} F:\{M\}=F \vee(0:\{M\})=F,
$$

a contradiction. Hence $\{M\}$ is principal in $\mathscr{L}_{M}$. It now follows by [7] that $\mathscr{L}$ is distributive and representable.

Recall that a Noether lattice $\mathscr{L}$ satisfies the woak union condition if, given elements $A, B$ and $C$ such that $A \not \equiv B$ and $A \not C$, it follows that there exists a principal element $E \leqq A$ such that $E \$ B$ and $E \nsubseteq C$. This concept was used in [7] to characterize the distributive Noether lattices which are representable. It is easy to see that if $\mathscr{L}$ is a Noether lattice which satisfies the weak union condition, then every localization $\mathscr{L}_{M}$ has the (weaker) property that, given primes $P_{1}, \cdots, P_{k}$ and an element $A$ such that $A \not P_{i}(i=1, \cdots, k)$, there exists a principal element $E \leqq A$ such that $E \$ P_{i}(i=1, \cdots, k)$. We say that a Noether lattice with this latter property satisfies the union condition on primes.

THEOREM 3.2. Let $\mathscr{L}$ be a distributive Nosther lattice such that, for every maximal element $M, \mathscr{L}_{M}$ satisfies the unior condition on primes. If 0 has no embedded primes and if every maximal element is join-principal, then $\mathscr{L}$ is Noether-lattice-embeddable in the lattice of ideals of a Noetherian ring. 
Proof. Let $0=Q_{1} \wedge \cdots \wedge Q_{k}$ be a normal decomposition in which $Q_{i}$ is $P_{i}$-primary. And let $M$ be a maximal element of $\mathscr{L}$. If $M$ is a prime of 0 , then $M$ is a minimal prime. On the other hand, by Lemma 1.1, if $M$ is not a prime of 0 , then 0 is prime in $\mathscr{L}_{M}$. Hence, if we assume that $P_{1}, \cdots, P_{s}$ are nonmaximal primes and that $P_{s+1}, \cdots, P_{k}$ are maximal primes, we have that

$$
\mathscr{L} \cong \mathscr{L}\left|P_{1} \oplus \cdots \oplus \mathscr{L}\right| P_{s} \oplus \mathscr{L} / Q_{s+1} \oplus \cdots \oplus \mathscr{L} / Q_{k} .
$$

Then each of the summands $\mathscr{L} / P_{i}(i=1, \cdots, s)$ is isomorphic to the lattice of ideals of some Noetherian ring [8], and each of the summands $\mathscr{L} / Q_{i}(i=s+1, \cdots, k)$ is Noether-lattice-embeddable in the lattice of ideals of a Noetherian ring (Corollary 2.3). The conclusion is now immediate.

By the results of [9], it is easy to see that any Noether lattice of the type described in Theorem 3.2 has the property that every element has a unique normal decomposition. On the other hand, a Noether lattice with this latter property is the direct sum of local Noether lattices with nilpotent maximal elements and one-dimensional Noether lattices in which 0 is prime [9]. These observations lead to the following, the proof of which is similar to the proof of Theorem 3.2:

THEOREM 3.3. Let $\mathscr{P}$ be a Nosther lattice in which each maximal element is join-principal. Then the following are equivalent:

(i) Each element has a unique normal decomposition.

(ii) Satisfies the union condition on primes and 0 has no embedded primes.

(iii) $\mathscr{L}$ is the (finite) direct sum of Noether lattices with principal maximal elements and local Nosther lattices with nilpotent maximal elements.

If, in addition, $\mathscr{L}$ is distributive, then each of the above implies that $\mathscr{C}$ is Noether-lattice-embeddable in the lattice of ideals of a Noetherian ring.

\section{REFERENCES}

1. K. P. Bogart, Structure theorems for regular local Noether lattices, Michigan Math. J., 15 (1968), 167-176.

2. - Distributive local Noether lattices, Michigan Math. J., 16 (1969), 215-223.

3. - Idempotent Noether lattices, Proc. Amer. Math. Soc., 22 (1969), 127-128.

4. - - Nonimbeddable Noether lattices, Proc. Amer. Math. Soc., 22 (1969), 129133.

5. R. P. Dilworth, Abstract commutative ideal theory, Pacific J. Math., 12 (1962), 481-498.

6. E. W. Johnson, A-transforms and Hilbert functions in local lattices, Trans. Amer. Math. Soc., 137 (1969), 125-139. 
7. E. W. Johnson, and J. P. Lediaev, Representable distributive Noether lattices, Pacific J. Math., 28 (1969), 561-564.

8. - - Join-principal elements and the Principal Ideal Theorem, Michigan Math. J., to appear.

9. —, - and J. A. Johnson, Structure and embedding theorem for unique normal decomposition lattices, Fund. Math., to appear.

Received February 17, 1970.

The University of Iowa 


\section{PACIFIC JOURNAL OF MATHEMATICS}

\section{EDITORS}

H. SAMELSON

Stanford University

Stanford, California 94305

C. R. Новву

University of Washington

Seattle, Washington 98105
J. DUGundJI

Department of Mathematics

University of Southern California

Los Angeles, California 90007

RICHARD ARENS

University of California

Los Angeles, California 90024

\section{ASSOCIATE EDITORS}
E. F. BECKENBACH
B. H. NeumanN
F. WOLF
K. YOSHIDA

\section{SUPPORTING INSTITUTIONS}
UNIVERSITY OF BRITISH COLUMBIA
CALIFORNIA INSTITUTE OF TECHNOLOGY
UNIVERSITY OF CALIFORNIA
MONTANA STATE UNIVERSITY
UNIVERSITY OF NEVADA
NEW MEXICO STATE UNIVERSITY
OREGON STATE UNIVERSITY
UNIVERSITY OF OREGON
OSAKA UNIVERSITY
UNIVERSITY OF SOUTHERN CALIFORNIA
STANFORD UNIVERSITY
UNIVERSITY OF TOKYO
UNIVERSITY OF UTAH
WASHINGTON STATE UNIVERSITY
UNIVERSITY OF WASHINGTON
AMERICAN MATHEMATICAL SOCIETY CHEVRON RESEARCH CORPORATION TRW SYSTEMS
NAVAL WEAPONS CENTER 


\section{Pacific Journal of Mathematics}

\section{Vol. 37, No. $1 \quad$ January, 1971}

Gregory Frank Bachelis and Haskell Paul Rosenthal, On unconditionally

converging series and biorthogonal systems in a Banach space .........

Richard William Beals, On spectral theory and scattering for elliptic

operators with singular potentials .........................

J. Lennart (John) Berggren, Solvable and supersolvable groups in which every element is conjugate to its inverse ........................ 21

Lindsay Nathan Childs, On covering spaces and Galois extensions ..........

William Jay Davis, David William Dean and Ivan Singer, Multipliers and

unconditional convergence of biorthogonal expansions..............

Leroy John Derr, Triangular matrices with the isoclinal property ............

Paul Erdős, Robert James McEliece and Herbert Taylor, Ramsey bounds for

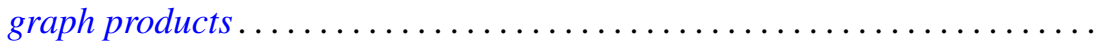

Edward Graham Evans, Jr., On epimorphisms to finitely generated

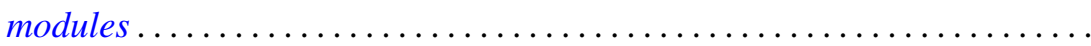

Hector O. Fattorini, The abstract Goursat problem ................. 51

Robert Dutton Fray and David Paul Roselle, Weighted lattice paths .........

Thomas L. Goulding and Augusto H. Ortiz, Structure of semiprime $(p, q)$

radicals ...........................................

E. W. Johnson and J. P. Lediaev, Structure of Noether lattices with join-principal maximal elements ....

David Samuel Kinderlehrer, The regularity of minimal surfaces defined over

slit domains

Alistair H. Lachlan, The transcendental rank of a theory. .

Frank David Lesley, Differentiability of minimal surfaces at the boundary ...

Wolfgang Liebert, Characterization of the endomorphism rings of divisible torsion modules and reduced complete torsion-free modules over complete discrete valuation rings....

Lawrence Carlton Moore, Strictly increasing Riesz norms.

Raymond Moos Redheffer, An inequality for the Hilbert transform ...

James Ted Rogers Jr., Mapping solenoids onto strongly self-entwined,

circle-like continua..........................

Sherman K. Stein, B-sets and planar maps ................... 217

Darrell R. Turnidge, Torsion theories and rings of quotients of Morita

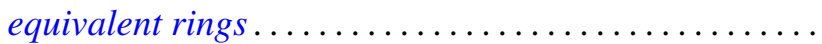

Fred Ustina, The Hausdorff means of double Fourier series and the principle of localization ................................

Stanley Joseph Wertheimer, Quasi-compactness and decompositions for arbitrary relations.

Howard Henry Wicke and John Mays Worrell Jr., On the open continuous images of paracompact $\check{C}$ ech complete spaces... 